\title{
Is bigger always better? The importance of cortical configuration with respect to cognitive
}

\section{ability}

Eero Vuoksimaa* a,b,c ${ }^{\text {atthew S. Panizzon }}{ }^{\text {a,b }}$, Chi-Hua Chen ${ }^{\text {a,b,d }}$, Mark Fiecas ${ }^{\text {a,b }}$, Lisa T. Eyler ${ }^{\text {e,a }}$, Christine Fennema-Notestine ${ }^{\mathrm{a}, \mathrm{d}}$, Donald J. Hagler Jr. ${ }^{\mathrm{d}}$, Carol E. Franz ${ }^{\mathrm{a}, \mathrm{b}}$, Amy J. Jak ${ }^{\mathrm{a}, \mathrm{f}}$, Michael J. Lyons $^{\mathrm{g}}{ }^{\text {, Michael C. Neale }}{ }^{\mathrm{h}}$, Daniel A. Rinker ${ }^{\mathrm{a}, \mathrm{d}, \mathrm{i}}$, Wesley K. Thompson ${ }^{\mathrm{a}}$, Ming T. Tsuang ${ }^{\mathrm{a}, \mathrm{b}}$, Anders M. Dale ${ }^{\mathrm{d}, \mathrm{j}}$, William S. Kremen* a,b,f

${ }^{a}$ Department of Psychiatry, University of California, San Diego, La Jolla, CA 92093, USA

${ }^{\mathrm{b}}$ Center for Behavioral Genomics Twin Research Laboratory, University of California, San Diego, La Jolla, CA 92093 USA

${ }^{\mathrm{c}}$ Department of Public Health, and Institute for Molecular Medicine (FIMM), University of Helsinki, Helsinki, Finland

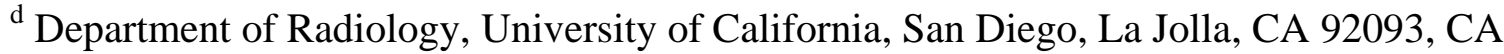

${ }^{\mathrm{e}}$ Mental Illness Research Education and Clinical Center, VA San Diego Healthcare System, San Diego, CA

${ }^{\mathrm{e}}$ Department of Radiology, University of California, San Diego, La Jolla, CA 92093, CA

${ }^{\mathrm{f}}$ Center of Excellence for Stress and Mental Health, VA San Diego Healthcare System, La Jolla, CA 92093, USA

${ }^{\mathrm{g}}$ Department of Psychological and Brain Sciences, Boston University, Boston, MA 02215, USA

${ }^{\mathrm{h}}$ Virginia Institute for Psychiatric and Behavioral Genetics, Virginia Commonwealth University School of Medicine, Richmond, VA 23220, USA

${ }^{\mathrm{i}}$ Imaging Genetics Center, Institute for Neuroimaging and Informatics, University of Southern California, Los Angeles, CA

${ }^{\mathrm{j}}$ Department of Neurosciences, University of California, San Diego, La Jolla, CA 92093, USA

Running title: Configuration of cortex and cognition

Word count

Abstract: 204

Main text (excluding abstract and references): 8715

Tables/figures: 2 Tables / 3 Figures

References: 55

Supplementary material: 9 Supplementary Tables \& 4 Supplementary Figures

* Corresponding authors:

Eero Vuoksimaa: Department of Public Health, University of Helsinki. P O Box 20

(Tukholmankatu 8 2B), FI-00014 University of Helsinki, Finland. E-mail:

eero.vuoksimaa@helsinki.fi

William S. Kremen: Department of Psychiatry, University of California, San Diego, 9500 Gilman

Drive (MC 0738), La Jolla, CA 92093, USA. E-mail: wkremen@ucsd.edu 


\begin{abstract}
General cognitive ability (GCA) has substantial explanatory power for behavioral and health outcomes, but its cortical substrate is still not fully established. GCA is highly polygenic and research to date strongly suggests that its cortical substrate is highly polyregional. We show in map-based and region-of-interest-based analyses of adult twins that a complex cortical configuration underlies GCA. Having relatively greater surface area in evolutionary and developmentally high-expanded prefrontal, lateral temporal, and inferior parietal regions is positively correlated with GCA, whereas relatively greater surface area in low-expanded occipital, medial temporal, and motor cortices is negatively correlated with GCA. Essentially the opposite pattern holds for relative cortical thickness. The phenotypic positive-to-negative gradients in our cortical-GCA association maps were largely driven by a similar pattern of genetic associations. The patterns are consistent with regional cortical stretching whereby relatively greater surface area is related to relatively thinner cortex in high-expanded regions. Thus, the typical "bigger is better" view does not adequately capture cortical-GCA associations. Rather, cognitive ability is influenced by complex configurations of cortical development patterns that are strongly influenced by genetic factors. Optimal cognitive ability appears to be driven both by the absolute size and the polyregional configuration of the entire cortex rather than by small, circumscribed regions.
\end{abstract}

Keywords: cortical surface area, cortical thickness, general cognitive ability, twin research, neurodevelopment 


\section{Introduction}

There is a long history of scientific curiosity about the neural underpinnings of individual differences in intelligence or general cognitive ability (GCA). Elucidating those brain-behavior relationships, including their genetic and environmental underpinnings, is important for understanding normal and pathological development and aging, and neuropsychiatric disorders.

Studies of associations between GCA and neocortical (hereafter referred to as cortical) gray matter size are growing in number. There are more studies of cortical thickness (CT) than of cortical surface area (SA). Studies of CT have been mixed, with reports of positive, negative, and no associations with GCA (Vuoksimaa et al., 2015). Although there are fewer studies of SA-GCA relationships, those have consistently shown significant positive SA-GCA associations (Vuoksimaa et al., 2015). Although there is some evidence in support of the predominant view that "bigger is better" when it comes to cortical-GCA associations, it seems likely that the cortical underpinnings of GCA are not so simple. A fundamental conundrum, for example, is the fact that men do not have higher average GCA than women despite having larger brains and larger cortex. Our view is that we need to think in terms of complex configurations of SA and CT in order to develop a more complete picture of the cortical underpinnings of GCA. At a minimum, this would seem to require combined examination of both SA and CT (see Schnack et al. 2015; Vuoksimaa et al. 2015), but that has been rare in studies of cortical-GCA associations.

At the global level, SA and CT are genetically independent (Panizzon et al., 2009), and SA appears to be the primary driver of the phenotypic and genetic association between cortex size and GCA (Vuoksimaa et al., 2015). However, there is also evidence that there are some, generally inverse SA-CT associations in some subregions (Panizzon et al., 2009). The relationship between regional SA and CT may be affected by the phenomenon of cortical stretching whereby cortical thinning is presumed to be caused by regional areal expansion. This phenomenon is seen throughout adulthood (Hogstrom, Westlye, Walhovd, \& Fjell, 2013) and is most pronounced in 
some prefrontal regions (Hogstrom et al. 2013; Panizzon et al., 2009); also when looking at the relative SA and CT (Winkler et al., 2010). Further evidence for the importance of looking at both relative SA and relative CT comes from studies of gyrification (Tallinen, Chung, Biggins \& Mahadevan, 2014; Razavi, Zhang, Liu \& Wang, 2015). Gyrification of the cortex, an important characteristic of the human brain, is positively correlated with SA but negatively with CT (Hogstrom et al., 2013). Recent work suggests that gyrification patterns are a function of relative cortical expansion and relative thickness (Tallinen et al., 2014). In the presence of tangential expansion, thinner cortex buckles and folds more easily than thicker cortex, which results in more gyrification (Zilles et al., 2013). In short, studying both cortical SA and CT is needed for greater understanding of brain morphometry and its behavioral correlates such as cognitive ability.

Both animal and human studies have demonstrated an anterior-posterior (A-P) gradient of genetic effects on cortical areal expansion. The same genes that cause anterior SA expansion also cause posterior contraction and vice versa (Bishop, Rubenstein, \& O'Leary, 2002; Chen et al., 2011; Chen et al., 2012; Chen et al., 2013; O'Leary, Chou, \& Sahara, 2007). We have also demonstrated that, relatively orthogonal to the A-P gradient of areal expansion, there is a dorsal-ventral (D-V) gradient of genetic influences on CT in the human brain indicating that the same genetic effects that make cortex relatively thicker in dorsal regions also make cortex relatively thinner in ventral regions and vice versa (Chen et al., 2013). In animal and human studies, these gradients from positive to negative correlations are observed only after global effects are taken into account, i.e., total SA and mean CT, respectively. Otherwise, the gradients simply shift from more strongly positive to less strongly positive: i.e., the same genetic effects that cause SA expansion in one region also cause SA expansion in other regions, and the same genetic effects that make cortex thicker in one region also make cortex thicker in other regions (Eyler et al., 2012). In order to elucidate relative regional effects, it is necessary to examine regional values in the context of global 
size. However, studies of the relationship between GCA and either SA or CT have not accounted for total SA or mean CT (reviewed by Vuoksimaa et al., 2015).

One recent finding regarding SA is the observation that better visuo-spatial reasoning ability was associated with greater areal expansion in prefrontal, lateral temporal, and inferior parietal cortices (Fjell et al., 2015). The authors pointed out that these are regions that have undergone the greatest expansion during evolution and human postnatal development. Indeed, it seems that the highly non-uniform areal expansion of the cortex follows the same pattern across species (humans versus non-human primates) and within human development (Chaplin, Yu, Soares, Gattass, \& Rosa, 2013; Fjell et al., 2015; Hill et al., 2010). Moreover, in humans the relatively highexpanded cortical regions also tend to be lightly and later myelinated, whereas relatively lowexpanded regions tend to include regions that are more heavily and early myelinated (Glasser \& Van Essen, 2011). Highly-expanded/lightly-myelinated regions include prefrontal, lateral temporal and inferior parietal cortices; relatively low-expanded/heavily-myelinated regions include occipital and medial temporal cortices as well as regions in and around the central sulcus (Fjell et al., 2013; Glasser \& Van Essen, 2011; Hill et al., 2010).

GCA is highly polygenic trait (Davies et al., 2014) and increased GCA has been positively selected in human evolution (Joshi et al. 2015). The patterning of cortical SA in humans also differs as a function of regions that are more strongly influenced by single nucleotide polymorphisms in more versus less evolutionarily conserved regions of the genome (Chen et al. 2015). However, the links among patterns of expansion/myelination, GCA, SA and CT have not yet been examined in combination, particularly with respect to genetic influences. In the current study, we took the novel approach of examining cortical maps of the relationship between GCA and both CT and SA while taking global size into account. Specifically, in line with the animal literature and our own earlier work, we scaled regional SA in relation to total SA and regional CT in relation to mean $\mathrm{CT}$ in order to investigate if the configuration of high-expanded and low-expanded regions 
relative to global size is related to GCA. We then statistically compared these maps to maps of regions with high- versus low-expansion/light- versus heavy-myelination. Finally, we used the power of our twin design to examine the contribution of genetic and environmental factors in the observed cortical-GCA associations.

We hypothesized that cortical-GCA relationships would be determined by the configuration of the cortex such that we would observe both positive and negative correlations in relation to global effects of total SA and mean CT when examining SA and CT, respectively. Thus, cortical-GCA relationships would not simply be consistently positive (i.e., "bigger is better"). Specifically, we hypothesized that: 1) the gradients from positive to negative correlations between relative SA and GCA would be consistent with the non-uniform cortical expansion patterns across evolution and human development (i.e., high-expanded regions having positive correlation with GCA and low-expanded regions having negative correlations with GCA); 2) relatively thinner cortex in high-expanded regions would be associated with better GCA; and 3) genetic factors would play a significant role in regional cortical-GCA associations.

In short, the current evidence of A-P and D-V gradients (Chen et al., 2011, 2013) and of the dynamics between relative SA and CT in the configuration of the cortex (Tallinen et al., 2014) suggests that investigating relative SA and CT in combination can shed light onto fundamental characteristics of cortical development. However, no studies have used the approach of looking at both relative SA and CT in the context of cognitive abilities. We use the term relative regional effects to indicate associations based on regional values relative to global size (e.g., the ratio of the $\mathrm{SA}$ at a vertex or a region of interest $[\mathrm{ROI}]$ to total $\mathrm{SA})$. 


\section{Materials and Methods}

\section{Participants}

Brain imaging and cognitive data at ages 51-60 were obtained for 534 men from the Vietnam Era Twin Study of Aging (VETSA 1: 2002-2008) (Kremen et al., 2013). Of those, 513 had analyzable MRI data for creating continuous cortical maps: 130 monozygotic (MZ); 96 dizygotic (DZ) twin pairs; and 61 unpaired individual twins. DNA-based zygosity was available for $92 \%$ of the pairs; for others it was determined by questionnaire and blood group. The VETSA sample is representative of U.S. men in their age range based on sociodemographic and health characteristics (Kremen et al., 2006; Schoenborn \& Heyman, 2009). All had prior military service, but most (78\%) were not exposed to combat (Eisen, True, Goldberg, Henderson, \& Robinette, 1987; Henderson et al., 1990).

Data were collected at two sites: University of California, San Diego and Boston University. Brain imaging in Boston was done at the Massachusetts General Hospital. All participants gave written informed consent to participate. The study was approved by the Institutional Review Boards of the participating universities.

\section{GCA measure}

GCA was measured with the Armed Forces Qualification Test, which is a 50-minute paperand-pencil test covering four domains of cognitive function: verbal ability (synonyms); arithmetic ability; spatial processing (mentally folding or unfolding boxes); and reasoning about tools and mechanical relationships. It has a high correlation $(\mathrm{r}=.84)$ with Wechsler IQ, and has a test-retest reliability of .74 over 35 years (Bayroff \& Anderson, 1963; Lyons et al., 2009; McGrevy, Knouse, \& Thompson, 1974). GCA is well-validated construct both at phenotypic and genetic levels (Panizzon et al., 2014; see Deary, 2012 for a review).

Image acquisition and processing

Images were acquired on Siemens 1.5 Tesla scanners. Two sagittal $\mathrm{T}_{1}$-weighted MPRAGE 
sequences were employed with a $\mathrm{TI}=1000 \mathrm{~ms}, \mathrm{TE}=3.31 \mathrm{~ms}$, TR=2730ms, flip angle $=7$ degrees, slice thickness $=1.33 \mathrm{~mm}$, voxel size $1.3 \times 1.0 \times 1.3 \mathrm{~mm}$. To increase the signal-to-noise ratio, the two $\mathrm{T}_{1^{-}}$ weighted MPRAGE acquisitions were rigidly registered to each other to account for head motion between scans and then averaged. The FreeSurfer software package (version 3) was used to reconstruct the cortical surfaces for each brain (Dale, Fischl, \& Sereno, 1999; Fischl, Sereno, \& Dale, 1999; Fischl \& Dale, 2000). Control point setting and manual editing were performed as necessary, based on standard objective editing rules of FreeSurfer developers, as described in Kremen et al. (2010). CT was calculated at each surface location as the shortest distance between the gray-white boundary and pial surfaces (Fischl \& Dale, 2000). Maps of CT for individual subjects were resampled into a common coordinate system using a spherical surface deformation method based on alignment of cortical folding patterns (Fischl et al., 1999). Maps of cortical SA were created by resampling a subject's gray-white boundary surfaces into a standard tessellation, such that the SA (in $\mathrm{mm}^{2}$ ) assigned to each point reflects the degree of expansion or contraction relative to the atlas (Chen et al., 2012; Joyner et al., 2009). A detailed description of the MRI image acquisition and processing in the VETSA study can be found in Eyler et al. (2012) and Kremen et al. (2010).

\section{Approach for testing the regional specificity}

We approached the regional specificity of cortical-GCA associations by mapping all individual vertex-GCA correlations throughout the cortex (about 150,000 vertices per hemisphere) so that we could visualize the gradients where the sign (positive versus negative) of the correlation changes. Most importantly, we wanted to compare these continuous unthresholded maps of areal expansion to those observed across human development and evolution. Here, an important step was to use relative rather than absolute regional measures. The procedure for taking into account the global effects of total SA and mean CT followed our earlier work that demonstrated the A-P and DV gradients for relative SA and relative CT, respectively (Chen et al., 2011; Chen et al., 2012; Chen 
et al., 2013). Specifically, we divided the surface area value at each location (vertex) by the total surface area (sum across all vertices) for each participant (Chen et al. 2011; Chen et al. 2012). This approach also follows the procedure implemented in animal studies of cortical gradients (Bishop et al., 2002; O’Leary et al., 2007). For thickness maps we subtracted the mean cortical thickness from the thickness at each vertex (Chen et al. 2013).

These relative measures are about scaling of cortical regions and they address a specific question of relative regional correlates of GCA. Adjusting for global size by using total SA and mean CT as covariates constitutes a different approach that would address a different question. Our relative SA and CT measures are different from each other because relative measures are acquired either by using the global measure for dividing (for SA) or subtracting (for CT). In addition to the high-expanded CT - low-expanded CT measure, we also used (high-expanded CT low-expanded CT) / mean CT in the ROI-based analysis. This latter scaled score corresponds to the SA difference score in the ROI-based analysis. As such, both explicitly measure the respective CT and SA gradients within individuals. In some analyses we subtracted mean CT from regional CT, whereas in others, we further divided that difference score by mean CT. However, we note that in correlational analyses these two measures are virtually the same; their close linear relationship is shown in Supplementary Figure S1.

To elucidate regional differences, many studies control for global volume measures (intracranial, whole brain, or partial gray matter). Whether the approach is to use a covariate, a ratio measure, or a difference score, these global measures ignore the fact that individual differences in cortical volume are largely attributable to variability in SA rather than CT (Im et al., 2008; Pakkenberg \& Gundersen, 1997). For example, controlling for total brain volume or intracranial volume means that adjusting for global effects will be very different in the context of SA or CT. However, if global size is not taken into account, it is not known if the associations reflect relative regional effects. Because using volume as the global measure would mean very different things for 
SA and CT (Ecker et al., 2013; Vuoksimaa et al., 2015; Wierenga, Langen, Oranje, \& Durston, 2014), we used global measures of 2-dimensional total SA and 1-dimensional mean CT-rather than a 3-dimensional volume measure - when mapping the regional SA and CT correlates of GCA, respectively. In addition to unthresholded continuous maps, we also created uncorrected p-value maps of all correlations that were significant at the level of $p<.05$ to explore the most robust correlations. This uncorrected threshold was selected because global measures of total SA and mean CT are highly correlated with regional SA and CT, respectively (Eyler et al., 2012); thus, making the use of these global measures as described in the preceding paragraph provides a robust adjustment in the search for relative regional specificity.

Finally, in addition to vertex-wise maps, we also used a ROI approach whereby we divided cortex into two ROI sets (Fig. 1). These ROI sets represent cortical regions that are high versus low expanded and were created by using 33 predefined ROIs (Desikan et al. 2006). If we control for vertex-wise multiple testing in the map-based analyses, we are substantially underpowered to detect differences between high- and low-expanded regions. Indeed, no correlations survived after correcting for multiple testing in the vertex-wise analyses (see Discussion). Thus, the primary purpose of the ROI-based analyses was to provide quantitative/statistical validation for differing patterns of association in high- and low-expanded regions that may be suggested by the map-based analyses.

Based on work by Hill et al. ( 2010), Glasser and Van Essen (2011), Chaplin et al. (2013), Amlien et al. (2014), Fjell et al. (2014), and Fjell et al. (2015) we created one ROI set that includes regions that are relative highly expanded both across evolution and human development compared to the rest of the cortex. The other, complementary ROI set included the remaining regions, which are relatively less expanded. In addition, our ROI sets also correspond to myelination maps whereby high-expanded regions are generally more lightly and late myelinated than the more heavily and early myelinated low-expanding cortex (Glasser and Van Essen, 2011; 
Amlien et al. 2014). We calculated a difference score between SA of the high-expanded ROI set and SA of the low-expanded ROI set and divided that difference score by total SA. As described earlier, we calculated difference scores for CT in two ways. These measures are indices of the strength of a potential gradient in size measures between high- and low-expanding regions of the brain in a given individual, and can be understood as a basic measure of the spatial configuration of $\mathrm{SA}$ or $\mathrm{CT}$ in that person.

\section{Statistical analyses}

Variance of a phenotype in the twin design can be modeled as being due to the following influences: additive genetic (A), non-additive/dominance genetic (D), common environmental (C), and unique environmental (E) influences (Neale \& Cardon, 1992). Classical twin models are commonly referred to as ACE or ADE models. With data from reared together pairs of twins, $\mathrm{C}$ and D effects cannot be estimated at the same time because the model would not be identified (Neale \& Cardon, 1992). Here we used ACE models. MZ twins correlate 1.0 with respect to A effects because they generally share $100 \%$ of their genes. DZs correlate 0.5 because, on average, they share $50 \%$ of their segregating genes. $\mathrm{C}$ is defined as environmental factors that make twins similar and correlate 1.0 both in MZs and DZs. E is defined as environmental factors that make twins different from one another (including measurement error), thus being uncorrelated within twin pairs.

The univariate model is easily extended to a multivariate scenario in which sources of covariance can also be examined. We used these models to compute phenotypic $\left(r_{p}\right)$, genetic $\left(r_{g}\right)$, and unique environmental $\left(\mathrm{r}_{\mathrm{e}}\right)$ correlations between GCA and SA or CT. We did not calculate common environmental correlations because the $\mathrm{C}$ estimates for the cortical measures were near zero. Genetic correlations index shared genetic variance between phenotypes. The genetic correlation is an index of only the shared genetic variance rather than the total phenotypic variance. 
Unique environmental correlations are analogous for unique environmental variance. All correlations were parametric.

Models were fit via the maximum likelihood-based structural equation modeling software Mx (Neale, Boker, Xie, \& Maes, 2004). ACE models were chosen as starting point. Accumulated evidence suggests that the contribution of C effects is very small for cortical phenotypes, and fixing $\mathrm{C}$ effects to zero in the models for CT or SA showed that AE models usually provided the best fits to the data (see Vuoksimaa et al. 2015). Genetic effects estimated in these AE models refer to broad-sense heritability, i.e., the proportion of phenotypic variance accounted for by the combined effect $(\mathrm{A}+\mathrm{D})$ of all genetic influences. Although it is possible that ADE models could have provided a good fit to the data, they would not have added useful information because we were underpowered to differentiate A and D effects. An ACE model was used for the GCA variable. Although the $\mathrm{C}$ variance was nonsignificant, in our view the estimate of .16 was too large to drop from the model. For genetic maps, models were fit using the variance-covariance matrices of cortical measures from paired $\mathrm{MZ}$ and $\mathrm{DZ}$ twins, with each location treated as an independent and continuous variable. The effects of age and scanner were regressed out of the cortical measures prior to analysis.

ROI-based analyses were performed with Mx software with raw data option by using a trivariate model with GCA, relative SA difference score and relative CT difference score as variables of interest. In line with the map-based analyses, the effects of age and scanner were regressed out of the cortical measures prior to analysis. The difference scores were then standardized to z-scores. We have previously used the same trivariate model approach when analyzing the global size measures of total SA and mean CT and their relationships with GCA (Vuoksimaa et al., 2015). We began with trivariate models that include GCA, SA, and CT. We refer to this trivariate Cholesky decomposition as the "ACE-ACE-ACE" Cholesky. This implies that the models include the $\mathrm{A}, \mathrm{C}$, and $\mathrm{E}$ variance components for each variable. Reduced models in 
which particular components were set to zero (e.g., "ACE-AE-AE") were then tested relative to these full Cholesky decompositions.

Model comparisons were based on the likelihood-ratio $\chi^{2}$-test, which is calculated as the change in $-2 \log$ likelihood ( $-2 \mathrm{LL}$ ) from the ACE-ACE-ACE Cholesky to the reduced model, and is distributed as a $\chi^{2}$ with degrees of freedom equal to the difference in parameters between the models. Nonsignificant $p$-values $(>0.05)$ indicate that the reduced model does not yield a significant change in the model fit and therefore provides an essentially equally good fit to the data while using fewer parameters. These models compute the phenotypic, genetic, common environmental, and unique environmental correlations between GCA, SA ROI and CT ROI. In sub-models, we tested if the phenotypic, genetic and environmental correlations could be constrained to be equal. These models directly tests if the GCA-SA and GCA-CT correlations differ from each other (i.e., is the pattern of cortical-cognition associations different for SA and CT). Significant $p$-values in these models indicate that constraining phenotypical, genetic or environmental correlations for GCA-SA and GCA-CT to be equal results in a poor fit of the model to the data.

\section{Measures for additional analyses}

We performed additional analyses to test the specificity of our findings, and to address the possibility that the results were simply an artifact of scaling for global effects. For example, controlling for global effects with only two ROI sets raises the question of whether the two will always be negatively correlated mirror images of one another.

To test whether the results of ROI-based analyses were specific to high- versus lowexpanded regions, we also created "balanced" cortical ROI sets (see Supplementary Fig S2) in which each had approximately equal proportions of high-and low-expanded regions. The ROI set referred to as "A" included 8 high-expanded and 9 low-expanded regions. The ROI set referred as "B" comprised the remaining cortex including 7 high-expanded and 9 low-expanded regions. Put another way, each had a nearly 50-50 split of high- and low-expanded regions: $53 \%$ of all high- 
expanded and 50\% of all low-expanded Desikan et al. ROIs in "A"; 47\% and 50\%, respectively, in "B." (see Supplementary material).

In addition to GCA, we also investigated the association between height and mental rotation ability with relative SA and CT in high- versus low-expanded ROI sets. Like GCA, both height and mental rotation ability are positively correlated with total SA. Mental rotation ability is another cognitive phenotype, and although correlated with GCA (like all cognitive abilities), it is considered as a specific cognitive domain which, according to a meta-analysis by Zacks (2008), is not expected to show a stronger association in the high-expanded ROI set compared to the lowexpanded ROI set. Mental rotation was assessed with the Card Rotations Test (Ekstrom et al., 1976). This test consists of 20 trials. Each trial has a particular irregular shape and participants must determine whether each of 8 pictures of the same shape have been rotated or would have to have been turned over. The score was the total number correct. Height was chosen as noncognitive phenotype, though it is also positively correlated with GCA (e.g., Marioni et al., 2014). Height was assessed in stocking feet with a stadiometer and rounded to the nearest half inch.

\section{Results}

Relationships between GCA and absolute areal expansion and thickness

Continuous maps of absolute regional cortical-GCA associations unadjusted for global size indicated that phenotypic correlations were positive at each vertex for the SA-GCA and for CTGCA associations (Fig. 2A). These positive associations were driven by genetic correlations, which indicate the extent to which the same genetic factors influence different phenotypes. Genetic correlations were positive at every vertex for the SA-GCA associations and over $99 \%$ of vertices for CT-GCA associations (Fig. 2B). Genetic correlations were more robust between SA and GCA than those between CT and GCA. The p-value maps of genetic correlations show that many more SAGCA than CT-GCA correlations were statistically significant (Fig. 2C). 
Topography of relative areal expansion-GCA correlations

The phrase "relative regional areal expansion" denotes regional SA measures in relation to total SA. As predicted, the pattern of cortical-GCA associations was dramatically different when examined in relation to global size. We now observed gradients in which corticalGCA correlations changed from positive to negative. Phenotypic correlations between GCA and relative areal expansion at each location were positive in prefrontal, lateral temporal, and inferior lateral parietal regions (Fig. 3A). In contrast, negative correlations emerged mainly in the region around the central sulcus and in the occipital, superior parietal, and medial temporal regions (Fig. $3 A$ ). According to the $\mathrm{p}$-value maps, the most robust positive SA-GCA phenotypic correlations were bilaterally in dorsolateral prefrontal regions and in middle and inferior temporal gyri in the lateral temporal cortex (Fig. 3B). The most robust negative correlations were around the central sulcus and paracentral lobule and in the posterior cingulate cortex in the right hemisphere, and in a small posterior region of left medial orbitofrontal cortex (Fig 3B)

Genetic correlation maps were generally similar to those seen at the phenotypic level. Positive genetic correlations were observed in prefrontal, lateral temporal, and inferior parietal regions whereas the genetic correlations were negative in central, superior parietal, medial temporal, and occipital regions (Fig. 3C). According to p-value maps, most robust positive SAGCA genetic correlations were observed both in the dorsolateral prefrontal and lateral temporal regions in the right hemisphere, and in a small prefrontal region in the left hemisphere (Fig. $3 D$ ). Paralleling the phenotypic map, the most robust negative genetic correlations were seen in the superior portion of the central sulcus in the right hemisphere, but also bilaterally in medial temporal lobe (Fig. 3D).

In the unique environmental correlation maps, correlations were lower than in the genetic correlation maps and they did not resemble maps seen at the phenotypic level; none of the environmental correlations were significant at the $\mathrm{p}<.05$ level (Supplementary Fig. S3). 


\section{Topography of relative CT-GCA correlations}

The term relative regional CT denotes regional CT measures in relation to overall mean CT (i.e., CT of each vertex - mean CT). Negative phenotypic CT-GCA correlations in prefrontal regions changed to positive correlations at the posterior regions of prefrontal cortex around the precentral sulcus. Posterior to the post-central sulcus, relative CT-GCA correlations were again negative in the parietal and occipital lobes. Correlations were positive in anterior temporal regions, shifting to negative in more posterior temporal cortex (Fig. 3A). According to the p-value maps, the most robust positive CT-GCA correlations were around the central sulcus bilaterally (on both the lateral and medial surfaces); the most robust negative correlations were seen bilaterally in prefrontal regions, specifically in lateral and medial orbitofrontal cortices, but also in the occipital lobe in the right hemisphere (Fig. 3B).

The genetic CT-GCA correlation maps were mostly parallel to the phenotypic maps (Fig. 3C), especially in the prefrontal regions (lateral and medial orbitofrontal cortices, and anterior cingulate). P-value maps showed that the most robust negative CT-GCA genetic associations were in regions of prefrontal cortex, but also in the occipital lobe, especially in the right hemisphere; the most robust positive CT-GCA genetic associations were detected around the central sulcus in the right hemisphere of (Fig. 3D). Environmental correlation maps did not resemble maps seen at the phenotypic level, and the environmental correlations were nonsignificant (Supplementary Fig. S3). Relative difference between high- and low-expanded cortex: Region-of-interest (ROI)-based analyses

We selected ROI sets from the Desikan parcellations (Desikan et al., 2006) in FreeSurfer that most closely corresponded to high-low-expanded/lightly-heavily-myelinated regions based on published reports (Hill et al., 2010; Glasser and Van Essen, 2011; Chaplin et al., 2013; Amlien et al., 2014epub; Fjell et al. 2014epub; Fjell et al. 2015) (Fig. 1). Selection was blind to SA or CT correlations with GCA. SA of the high- and low-expanded ROI sets were $723 \mathrm{~cm}^{2}$ (SD=64) 
and $901 \mathrm{~cm}^{2}(\mathrm{SD}=72)$, representing $45 \%$ (range $42-48 \%$ ) and 55\% (range 52-58\%) of the cortex, respectively. We compared these two ROI sets with relative cortical difference scores: ([highexpanded SA - low-expanded SA]/total SA); and (mean CT of high-expanded regions - mean CT of low-expanded regions). Results indicated that the SA of the high-expanded ROI set was on average $11 \%$ (range $4-16 \%$ ) smaller than the SA of the low-expanded ROI set. Mean CT was $2.07 \mathrm{~mm}$ $(\mathrm{SD}=0.09)$ in the high-expanded ROI set and $1.91 \mathrm{~mm}(\mathrm{SD}=0.08)$ in the low-expanded ROI set, yielding an average of $0.16 \mathrm{~mm}$ (range $0.04-0.29 \mathrm{~mm}$ ) thicker cortex in high-expanded ROI set compared to low-expanded ROI set.

The heritability of GCA was $0.62(95 \%$ CI: $0.35 ; 0.82)$. Both relative cortical difference scores also had substantial heritability: 0.42 (95\% CI: 0.29 ; 0.54$)$ for SA and 0.71 (95\% CI: $0.62 ; 0.78)$ for CT. A trivariate genetic analysis of the relative SA and CT difference scores and GCA indicated that at the phenotypic level, the relative SA difference score was significantly positively associated with GCA $(r=0.15 ; 95 \%$ CI: $0.05 ; 0.24$; see Supplementary Figure S4 for a scatter plot) but the relative CT difference was significantly negatively related to GCA ( $\mathrm{r}=-0.11$; 95\% CI: $-0.20 ;-0.01)$. Specifically, individuals with higher GCA were those whose SA was relatively greater in the high-expanding compared to low-expanding regions and whose cortex was relatively thinner in high-expanding compared to low-expanding regions. Similarly, at the genetic and environmental levels, the correlations were opposite in sign: SA-GCA $\left(r_{g}=0.26\right.$; $95 \%$ CI: 0.06 ; 0.50 and $r_{e}=0.03 ; 95 \%$ CI: $\left.-0.13 ; 0.19\right)$ and CT-GCA $\left(r_{g}=-0.11 ; 95 \%\right.$ CI: $-0.27 ; 0.05$ and $r_{e}=-0.15$; 95\% CI: -0.31; 0.02) (Model fit statistics in Table 1, correlations in Table 2).

The phenotypic and genetic SA-GCA and CT-GCA correlations were significantly different from one another as indicated by the fact that constraining them to be equal resulted in significant reductions in model fit (Models 3 and 4 in Table 1, respectively). However, environmental SA-GCA and CT-GCA correlations could be constrained to be equal (Model 5 in Table 1). Finally, derived from Model 2 in Table 1, there were significant negative phenotypic and 
genetic correlations between the relative SA and CT difference scores: $r_{p}=-0.14$ (95\% CI: -0.23 ; $0.05)$ and $r_{g}=-0.21(95 \%$ CI: $-0.40 ;-0.02)$. The environmental SA-CT correlation was nonsignificant $r_{\mathrm{e}}=-0.06(95 \% \mathrm{CI}:-0.22 ; 0.10)$.

When using the version of the scaled CT difference score whereby the difference in high-expanded and low-expanded ROIs was divided by mean CT, the results were similar to the results with the simple difference between high-expanded and low-expanded ROIs presented above: CT-GCA r $=-0.12(95 \%$ CI: $-0.22 ;-0.03)$ (see Supplementary Tables S1 and S2 for model fitting statistics and correlations, respectively). With this CT difference score, the correlation between SA and CT was -..18.

\section{Relationship between GCA and balanced ROI}

SA of the "A" and "B" ROIs were $902 \mathrm{~cm}^{2}(\mathrm{SD}=74)$ and $722 \mathrm{~cm}^{2}(\mathrm{SD}=61)$ representing 56\% (range 53-59\%) and 44\% (range 41-47\%) of the cortex, respectively. Mean $\mathrm{CT}$ in the "A" ROI was $2.00 \mathrm{~mm}(\mathrm{SD}=0.09)$ and $1.95 \mathrm{~mm}(\mathrm{SD}=0.08)$ in the "B" ROI. Heritabilities for relative difference scores were 0.31 (95\%CI: 0.17 ; 0.45$)$ and 0.63 (95\%CI: $0.53 ; 0.72)$ for SA and CT, respectively. Relative SA and CT difference scores were not significantly correlated with each other: $r_{p}=-0.06$ (95\%CI: $\left.-0.15 ; 0.03\right)$. Constraining any of the SA-GCA and CT-GCA correlations to be equal did not result in a significant deterioration in model fit (Supplementary Table S3). None of those correlations were statistically significant (Supplementary Table S4). Next, we created four additional balanced ROI sets to reflect alternative divisions of the entire cortex. None of the difference scores of these ROI sets resembled the pattern observed with cortical division of high- versus low-expanded ROIs (Supplementary Tables S5 and S6).

Relative difference between high- and low-expanded cortex: associations with height and mental rotation ability

Relatively greater SA in high-expanded ROI was positively associated with height $r_{p}=$ 0.17 (95\%CI: 0.07; 0.26), but not with mental rotation ability $r_{p}=0.04$ (95\%CI: $\left.-0.05 ; 0.13\right)$. 
Relatively thinner cortex in high-expanded ROI was not associated with height $r_{p}=-0.03$ (95\%CI: $0.12 ; 0.07)$ or mental rotation ability $r_{p}=-0.09$ (95\%CI: $\left.-0.19 ; 0.004\right)$.

\section{Discussion}

Size Matters

We extended our finding that GCA was significantly associated with total SA rather than mean CT in this sample of middle-aged men (Vuoksimaa et al., 2015) by demonstrating that vertex-wise absolute SA and CT measures were positively correlated with GCA, and that those associations were stronger for SA than for CT. These findings are consistent with the fact that the volume of the human cortex is greater than in other primates, and that the cross-species volume expansion is more strongly related to greater areal expansion than to differences in cortical thickness (Changizi, 2001; Rakic, 2009). Moreover, in humans individual differences in cortical volume are largely attributable to variability in cortical surface area (Im et al., 2008; Pakkenberg \& Gundersen, 1997). These observations thus appear to be consistent with the idea that "bigger is better" when it comes to brain structure and cognitive ability. On the other hand, "bigger is better" cannot tell the entire story. For example, it cannot explain the fact that there are no sex differences in overall GCA despite consistent sex differences in human brain size.

\section{Configuration Also Matters}

An earlier report demonstrated the importance of high-expanded regions for visuospatial reasoning ability with analyses focused on positive correlations with SA unadjusted for global effects (Fjell et al., 2013). In our own prior work that did not involve any cognitive measures, we showed positive-to-negative gradients of genetic influences on SA and CT when accounting for global effects, with each having relatively orthogonal gradients (Chen et al., 2011; Chen et al., 2013). Here, by examining relative regional effects, we showed that there are complex patterns of both positive and negative SA-GCA associations rather than only positive associations. 
We also examined relative regional effects of CT in relation to GCA. As hypothesized, there was also a pattern of positive versus negative associations between GCA and relative CT, but one that contrasted sharply with that seen in SA-GCA associations. To a large extent there were inverse regional associations between SA and CT, and their regional correlations with GCA tended to be in opposite directions as well. For example, there were inverse SA and CT correlations for the GCAprefrontal cortex associations that were in line with the negative regional correlation between SA and CT. These relationships are consistent with the notion of cortical stretching, whereby increased regional SA is related to cortical thinning in the same region; this phenomenon has been reported to be most pronounced in prefrontal regions (Hogstrom et al., 2013). Note also that these corticalGCA patterns of gradients are rather different from the anterior-posterior and dorsal-ventral gradients of genetic influences that were examined without respect to any specific phenotype (Chen et al., 2011; Chen et al., 2013).

Tallinen et al. (2014) showed that at the global level gyrification patterns are a function of relative cortical SA and CT. They demonstrated that increased gyrification results from greater areal expansion, which is related to relatively thinner cortex; they argued that this effect is caused by non-uniform mechanical pressure. Controlling for estimated total intracranial volume or total brain volume, there were negative correlations between total SA and mean CT in our sample $(\mathrm{r}=-.23$ and $\mathrm{r}=-.32$, respectively; $p \mathrm{~s}<.001)$. The negative correlations between relative SA and CT demonstrate that it is important to look at relative size in addition to absolute size. It is well established that global absolute size of SA and CT are uncorrelated (Panizzon et al., 2009; Winkler et al., 2010), but our observations as well as those of Tallinen et al. show that with a given brain size relatively more cortical expansion is related to relatively thinner cortex.

The importance of interplay between cortical areal expansion and thickness in the cortical folding patterns suggests gyrification as an important cortical correlate of GCA in humans. Our work examining global size measures showed that although gyrification was positive related to 
GCA, this association was not evident when controlling for SA; in contrast, the SA-GCA association was evident when controlling for a gyrification index (Docherty et al., 2014). It is possible, however, that there may be regional associations. Interestingly, a study that controlled for intracranial volume, indicated positive and negative regional gyrification-cognition correlations in domains of working memory and executive functioning, respectively (Gautam et al., 2015).

Our results may raise the question of why other studies have not detected positive-tonegative gradients when examining cortical-GCA associations. We can think of at least three reasons. First, many studies have examined regional effects, but not in relation to global effects. Second, suboptimal global indices (e.g., height, cortical volume) have frequently been employed; these are problematic, in part, because CT is one-dimensional and SA is two-dimensional (Vuoksimaa et al., 2015; Wierenga et al., 2014). Our approach maintains consistency with this dimensionality. Finally, studies that simultaneously examine the relationship of cognition to both CT and SA are rare.

\section{Resemblance to Areal Expansion Maps of Human Development and Evolution}

Although cortical stretching may account for some of the inverse regional associations for SA and $\mathrm{CT}$, the predominant theme appears to be resemblance of our maps with areal expansion maps of human development and evolution and maps of myelination (Hill et al., 2010; Glasser and Van Essen, 2011; Amlien et al., 2014; Fjell et al. 2014; Fjell et al. 2015). Our results indicate that with respect to GCA, the nature of the inverse relationship between SA and CT (see also Tallinen et al. 2014) after accounting for global effects is a function of differences in high- and low-expanding regions. Consistent with our hypotheses, gradients of positive-to-negative SA-GCA correlations largely corresponded to the patterning of high- versus low-expanded/lightly- versus heavilymyelinated regions when scaled for total SA. GCA tended to be positively correlated with relative SA in high-expanded/lightly-myelinated regions and negatively correlated with relative SA in lowexpanded/heavily-myelinated regions. The strongest positive SA-GCA correlations were seen 
bilaterally in dorsolateral prefrontal cortex and in lateral temporal cortex. In contrast, negative SAGCA correlations were seen in regions that are low-expanded and heavily-myelinated. In contrast, positive CT-GCA correlations were seen mainly in low-expanded/heavily-myelinated central regions, and relatively thinner prefrontal cortex (a mostly high-expanded region) was related to higher GCA.

\section{Whole cortex approach and the polyregional nature of GCA}

We chose to examine unthresholded maps of correlations and thresholded p-value maps that were uncorrected for multiple comparisons in order to address our hypotheses about the patterns of association and resemblance to other continuous maps of expansion/myelination. That may raise questions as to the significance of the maps or whether SA and CT maps are truly different from one another. We already knew that global cortical size and total SA were positively related to GCA (Vuoksimaa et al., 2015). Here we sought to examine what underlies that global effect. We decided against the use of false discovery rate (FDR)-based p-value maps for several reasons. Using FDR is appropriate if one expects effects in circumscribed cortical regions, but — as in the studies we reviewed (Vuoksimaa et al., 2015) — the GCA-brain relationship is consistently found to be what we refer to as a polyregional phenomenon across the cortex. An FDR correction would not be consistent with our goal of mapping gradients of positive-to-negative cortical-GCA associations. We believe that mapping polyregional gradients is more biologically plausible than an expectation of only a few small, sharp, circumscribed regional peaks being related to GCA (as is suggested by the p-value maps). We also wanted to avoid common interpretative problems associated with FDR-based thresholded maps: confining interpretations to only the highest peaks of relationships can lead to false inferences of regional specificity not supported by the existing mapbased studies, or can result in generalization of the findings to surrounding areas which effectively nullifies the protection for multiple comparisons which prompted the use of FDR. Finally, continuous maps are most desirable for assessing genetic relationships of GCA with SA and CT 
because genetic patterning of the cortex does not have a perfect resemblance with any regional parcellation based on non-genetic information (Rimol et al., 2010; Chen et al., 2011; Chen et al., 2012).

Although the correlations with relative regional measures (relative to global size measures of total SA and mean CT) were small in magnitude, our maps did show regions where GCA is positively correlated with SA and negatively correlated with CT, or the reverse. Because each was significantly different from zero in the opposite direction, those correlations must be significantly different from one another. We note that after correcting for multiple comparisons, no correlations survived in continuous maps. On the other hand, our maps clearly show meaningful patterns in line with our prediction. In our view, the lack of significant result after correction for thousands of tests is analogous to the rarity of finding associations at genome-wide significance levels in genome-wide association studies. For highly polygenic phenotypes such as cortex and GCA (Davies et al., 2014; Chen et al., 2015), there are most likely large numbers of genes with effects that are too small to have genome-wide significance. However, approaches that include all significant genes or SNPs without correction for multiple testing are now being shown to be very useful in predicting cases versus controls (Escott-Price et al. 2015).

In addition, our ROI-based analyses fulfilled their primary purpose of providing strong validation of inferences drawn from the map-based analysis because they provide statistical confirmation of phenotypic and genetic patterns that are parallel and consistent with the map-based analyses. The ROI-based approximation of high-/low-expanded regions demonstrated that the SAGCA and CT-GCA associations were in opposite directions and significantly different from one another. The ROI-based analyses also allow for somewhat more specificity of interpretation compared to the map-based analysis. Specifically, with these analyses we showed the configuration of size measures within an individual (as indexed by their high- versus low-expanding proportional 
difference score) is a heritable phenotype that is related to GCA in different directions for SA and CT measures.

It should be noted that our use of global measures of total SA and mean CT in the context of regional SA and CT was about scaling; not adjusting for a global size covariate. We chose this approach because we were interested to study possible positive versus negative gradients of cortical-cognition associations. Scaling was a reasonable choice as we were interested to investigate if non-uniform stretching is related to cognitive ability. Earlier animal literature and our own work on genetic cortical gradients have used the same approach to scaling cortical surface area and cortical thickness. We acknowledge that for different questions about cortical-cognition associations, there are alternative ways to take global cortical measures of total SA and mean CT into account in regional analyses. Finally, regardless of the analytical strategy, we believe that using total SA and mean CT instead of cortical volume is the approach of choice when scaling or adjusting SA and CT, respectively.

Real findings or artifacts?

Controlling for global size measures of total SA and mean CT implies that relatively greater size in some regions is related to relatively smaller size in other regions. Hence, one might be tempted to argue that the shift to both positive and negative correlations in the map-based analyses is simply an artifact of accounting for global effects. For ROI-based analyses, with only two ROIs sets covering the entire cortex, one might also infer that the two must be negatively correlated (i.e., almost mirror images of one another). However, as shown in our additional analyses below (see also Supplementary material) these approaches do not necessarily imply that: 1) the positive versus negative correlations have to be significantly different from each other; or 2) the gradients of positive and negative associations follow the pattern of cortical expansion across evolution and human development. Regarding the latter point, even if accounting for global effects automatically results in a mix of positive and negative correlations, it is entirely uninformative as to 
where across the cortex those correlations will be. It is also worth noting that there is a substantial animal and human literature on cortical phenomena based on studies for which global effects are accounted (e.g., Chen et al., 2013).

To test whether the ROI-based results were an artifact of creating two ROI sets for the entire cortex, we created two new ROI sets that each had very similar proportions of high- and lowexpanded regions. Unlike the high- versus low-expanded ROI sets, none of the correlations were significant in the new analyses (Supplementary Tables S3, S4, Figure S2; see also Supplementary Tables S5 and S6).

In order to show that the inverse SA-GCA and CT-GCA relationships were not simply an artifact of correction for global measures of total SA and mean CT and the fact that relative SA and CT are negatively correlated in high-expanded regions, we also looked at the relative SA and CT difference scores in relation to two other phenotypes: height and a mental rotation ability (MRA). These analyses were conducted with the same high- versus low-expanded ROI sets as shown in Figure 1. All three phenotypes (GCA, height, and MRA) were positively correlated with total SA, and in every case relatively greater SA in the same high-expanded ROI set was related to relatively thinner cortex. However, each of these phenotypes had different patterns of associations with SA and CT (Supplementary Tables S7-S9). The fact that we observed different results in all of these additional analyses provides evidence that the observed relative SA-GCA and CT-GCA associations were not simply artifacts that arise from accounting for global effects or dividing the cortex into two ROI sets.

\section{Genetic and Environmental Influences}

We showed that the genetic correlation maps resembled the phenotypic maps, whereas there was no correspondence between phenotypic and environmental correlation maps. The phenotypic associations in the relative regional maps were driven primarily by shared genetic 
effects between SA and GCA. The ROI-based analyses served to confirm this interpretation, indicating that the association between relative SA and GCA was due to shared genetic effects, whereas the environmental correlation between these two was not significant. Our ROI-based results also indicated that the phenotypic correlations between relative CT and GCA were significantly different and opposite in sign from those between relative SA and GCA. Although both the genetic and environmental relative CT-GCA correlations were nonsignificant in the ROIbased analysis, the model in which the environmental SA-GCA and CT-GCA correlations were constrained to be equal did not yield a significant degradation of the model fit. In contrast, the genetic correlation between relative CT and GCA was significantly different and opposite in sign from the genetic correlation between relative SA and GCA.

\section{Limitations}

Our all male sample limits the generalizability of these results in females. We looked only at cross sectional SA-GCA and CT-GCA associations, whereas other studies have suggested that the timing of developmental cortical changes are also important for GCA (Shaw et al., 2006; Schnack et al., 2015). It will also be important to study how the configuration of SA and CT is related to specific cognitive abilities (e.g., episodic memory). Finally, a full understanding of structural brain-cognition relationships will ultimately require studying whole brain, including white matter, subcortical structures and cerebellum (see Ritchie et al., 2015). 


\section{Conclusions}

Our results complement, rather than contradict, findings regarding the relationship between absolute cortex size and GCA. Although "bigger is better" throughout the cortex when it comes to absolute size (Vuoksimaa et al., 2015), the primary new finding from our current analyses highlights the incompleteness of that explanation. The situation may be analogous to factor analytic studies of cognition in which there are consistent findings of a general factor plus specific ability factors that are independent of the general factor (Panizzon et al., 2014). Similarly, accounting for global effects revealed an additional dimension in the understanding of cortical-GCA associations, but both dimensions are important. Beyond the global "bigger is better" effect, the cortical underpinnings of GCA appear to be complex configurations that involve relatively larger size in some regions in conjunction with relatively smaller size in others. Although we could not examine sex differences in this all-male sample, the importance of relative cortical configurations may partly account for the lack of male-female differences in GCA despite overall cortical volume differences. Indeed, the potential ability to account for this sex difference conundrum suggests that this added dimension of configuration may even be more important than absolute size. This, of course, must be tested in mixed-sex samples.

These configurations are driven primarily by shared genetic factors, and they fit well with evolutionary and developmental maps of cortical areal expansion. More relative areal expansion of high-expanded regions (that are generally also late and lightly myelinated), but less relative areal expansion of low-expanded regions (that are generally also early and heavily myelinated) was associated with better GCA. The opposite pattern was observed in the relationship between CT and GCA.

These complex positive-to-negative gradient configurations of relative regional cortical correlates of cognition were only evident after accounting for global cortical effects (i.e., scaled for total SA/mean CT). In order to detect these gradient patterns for a highly polyregional 
phenotype such as GCA, it is important to examine the entire cortex without limiting analysis to a subset of regions, and to avoid arbitrary thresholds that might mask continuous gradient patterns. A more complete picture will also be obtained if analyses include both SA and CT; not being limited to one or the other is particularly important for genetically-informative studies because there are very different genetic influences on SA and CT (Panizzon et al., 2009). There has been a focus on the importance for human cognition of regions that have undergone high areal expansion in evolution and development (Hill et al., 2010; Fjell et al., 2015; Fjell et al, 2014), but our results show that CT is also an important component of cortical-GCA associations.

Both relatively greater SA and relatively thinner cortex in high-expanded regions were associated with higher GCA. Our results also indicated that when looking at relative regional SACT associations in a whole cortex approach, these two dimensions of cortex have shared genetic effects that account for the observed negative correlation. This demonstrates again that looking at relative SA and CT measures complements and extends analyses with absolute size measures, since our previous work indicated zero correlation between global SA and CT (Panizzon et al., 2009).

Both size and changes in SA and CT are important for brain morphology as suggested by recent work on the mechanisms behind cortical folding patterns (Tallinen et al. 2014; Razavi et al. 2015). Moreover, cortical stretching suggests that SA and CT are inversely related in some regions (Hogstrom et al., 2013). Although our results do not explicitly address the question of whether it was the combination of relatively greater SA and relatively thinner cortex in highexpanded regions that was associated with higher GCA, this interpretation (although speculative) would fit well with the idea of a relationship between non-uniform cortical expansion and relatively thinner cortex.

Brain function may, in part, be dependent on maintenance of a delicate balance in the interplay of factors affecting SA and CT in evolutionarily/developmentally older and newer regions (Fjell et al. 2014; Tallinen et al. 2014). As such, it is also possible that changes in cortical 
configurations might be more important for understanding cognitive decline, brain aging, and neuropsychiatric diseases than the more typical approach of focusing on which regions display the largest size reductions. Although studies of aging often focus on brain atrophy in particular regions, our results suggest that successful cognitive and brain aging might be more about one's particular pattern of atrophy such that there is relative maintenance of an optimal configuration.

In sum, both GCA and the structure of cortex have evolved as highly polygenic traits (Davies et al., 2014; Chen et al., 2015). Our study shows that individual differences in the complex cortical configuration of SA and CT are related to individual differences in overall cognitive ability, that cortical-GCA associations are driven by genetic effects, and that these associations are consistent with the patterns of cortical expansion across evolution and human development and negative associations between relative SA and CT. 


\section{Acknowledgements}

\section{Funding}

This work was supported by NIA grants R01 AG018386, AG022381, AG022982, AG050595 to WSK, R01 AG018384, AG050595 to MJL, K08 AG047903 to MSP, R01 GM104400 to WKT, and Academy of Finland grant 257075 to EV, and resources of the VA San Diego Center of Excellence for Stress and Mental Health Healthcare System. The Cooperative Studies Program of the US Department of Veterans Affairs provided financial support for development and maintenance of the Vietnam Era Twin (VET) Registry.

\section{Notes}

The content is solely the responsibility of the authors and does not necessarily represent official views of the NIH or the VA. Numerous organizations provided invaluable assistance in the conduct of this study, including: Department of Defense; National Personnel Records Center, National Archives and Records Administration; the Internal Revenue Service; National Opinion Research Center; National Research Council, National Academy of Sciences; the Institute for Survey Research, Temple University. We gratefully acknowledge the cooperation and participation of the members of the VET Registry and their families.

Anders Dale is a founder and holds equity in CorTechs Laboratories and also serves on its Scientific Advisory Board. The terms of this arrangement have been reviewed and approved by the University of California, San Diego, in accordance with its conflict of interest policies. No other authors have any conflicts of interest to declare. 


\section{References}

Amlien I, Fjell AM, Tamnes CK, Grydeland H, Krogsrud SK. et al. 2014. Organizing principles of human cortical development - thickness and area from 4 to 30 years: insights from comparative primate neuroanatomy. Cereb Cortex. doi:10.1093/cercor/bhu214.

Bayroff AG, Anderson AA. 1963. Development of armed forces qualification tests 7 and 8. (Technical Research Report No.1122). Arlington, VA: U.S. Army Research Institute.

Bishop KM, Rubenstein JL, \& O'Leary DD. 2002. Distinct actions of Emx1, Emx2, and Pax6 in regulating the specification of areas in the developing neocortex. J Neurosci. 22:7627-7638.

Changizi MA. 2001. Principles underlying mammalian neocortical scaling. Biological Cybernetics, $84: 207-215$.

Chaplin TA, Yu HH, Soares JG, Gattass R, Rosa MG. 2013. A conserved pattern of differential expansion of cortical areas in simian primates. J Neurosci. 33:15120-15125.

Chen CH, Peng Q, Schork AJ, Lo MT, Fan CC, Wang Y. et al. 2015. Large-scale genomics unveil polygenic architecture of human cortical surface area. Nat Commun. 6:7549. doi:

10.1038/ncomms8549.

Chen CH, Fiecas M, Gutierrez ED, Panizzon MS, Eyler LT, Vuoksimaa E. et al. 2013. Genetic topography of brain morphology. Proc Natl Acad Sci USA. 110:17089-17094. 
Chen CH, Gutierrez ED, Thompson W, Panizzon MS, Jernigan TL, Eyler LT. et al. 2012. Hierarchical genetic organization of human cortical surface area. Science. 335:1634-1636.

Chen CH, Panizzon MS, Eyler LT, Jernigan TL, Thompson W, Fennema-Notestine C. et al. 2011. Genetic influences on cortical regionalization in the human brain. Neuron. 72:537-544.

Dale AM, Fischl B, Sereno MI. 1999. Cortical surface-based analysis. I. segmentation and surface reconstruction. Neuroimage. 9:179-194.

Davies G, Tenesa A, Payton A, Yang J, Harris SE, Liewald D. et al. 2011. Genome-wide association studies establish that human intelligence is highly heritable and polygenic. Mol Psychiatry. 16:996-1005.

Deary IJ. 2012. Intelligence. Annu Rev Psychol. 63:453-482.

Docherty AR, Hagler DJ Jr, Panizzon MS, Neale MC, Eyler LT, Fennema-Notestine C, et al. 2015. Does degree of gyrification underlie the phenotypic and genetic associations between cortical surface area and cognitive ability? Neuroimage. 106:154-60.

Ecker C, Ginestet C, Feng Y, Johnston P, Lombardo MV, Lai MC. et al. 2013. Brain surface anatomy in adults with autism: The relationship between surface area, cortical thickness, and autistic symptoms. JAMA Psychiatry. 70:59-70. 
Eisen S, True W, Goldberg J, Henderson W, Robinette CD. 1987. The vietnam era twin (VET) registry: Method of construction. Acta Geneticae Medicae Et Gemellologiae, 36:61-66.

Ekstrom RB, French JW, Harmon HH. 1976. Manual for Kit of Factor-Referenced Cognitive Tests. Princeton, NJ: Educational Testing Service.

Escott-Price V, Sims R, Bannister C, Harold D, Vronskaya M, Majounie E, et al. 2015. Common polygenic variation enhances risk prediction for Alzheimer's disease. Brain 138(Pt 12):3673-84.

Eyler LT, Chen CH, Panizzon MS, Fennema-Notestine C, Neale MC, Jak A, et al. 2012. A comparison of heritability maps of cortical surface area and thickness and the influence of adjustment for whole brain measures: A magnetic resonance imaging twin study. Twin Res Hum Genet. 15:304-314.

Fischl B, Dale AM. 2000. Measuring the thickness of the human cerebral cortex from magnetic resonance images. Proc Natl Acad Sci USA. 97:11050-11055.

Fischl B, Sereno MI, Dale AM. 1999. Cortical surface-based analysis. II: Inflation, flattening, and a surface-based coordinate system. Neuroimage. 9:195-207.

Fjell AM, Amlien I, Sneve MH, Grydeland H, Tamnes CK, et al. 2014. The roots of alzheimer's disease: are high-expandingcortical areas preferentially targeted? Cereb Cortex. doi:10.1093/cercor/bhu055. 
Fjell AM, Westlye LT, Amlien I, Tamnes CK., Grydeland H, Engvig A, et al. 2015. Highexpanding cortical regions in human development and evolution are related to higher intellectual abilities. Cereb Cortex. 25:26-34

Gautam P, Anstey KJ, Wen W, Sachdev PS, Cherbuin N. 2015. Cortical gyrification and its relationships with cortical volume, cortical thickness, and cognitive performance in healthy mid-life adults. Behav Brain Res. 287:331-9.

Glasser MF, Van Essen DC. 2011. Mapping human cortical areas in vivo based on myelin content as revealed by T1- and T2-weighted MRI. J Neurosci. 31:11597-11616.

Henderson WG, Eisen S, Goldberg J, True WR, Barnes JE, Vitek ME. 1990. The vietnam era twin registry: A resource for medical research. Public Health Reports (Washington, D.C.: 1974), 105:368-373.

Hill J, Inder T, Neil J, Dierker D, Harwell J, Van Essen D. 2010. Similar patterns of cortical expansion during human development and evolution. Proc Natl Acad Sci USA. 107:13135-13140.

Hogstrom LJ, Westlye LT, Walhovd KB, Fjell AM. 2013. The structure of the cerebral cortex across adult life: Age-related patterns of surface area, thickness, and gyrification. Cereb Cortex. 23:2521-2530.

Im K, Lee JM, Lyttelton O, Kim SH, Evans AC, Kim SI. 2008. Brain size and cortical structure in the adult human brain. Cereb Cortex. 18: 2181-2191. 
Joshi PK, Esko T, Mattsson H, Eklund N, Gandin I, Nutile T. et al. 2015. Directional dominance on stature and cognition in diverse human populations. Nature. doi: 10.1038/nature14618. [Epub ahead of print]

Joyner AH, Roddey J, Bloss CS, Bakken TE, Rimol LM, Melle I. et al. 2009. A common MECP2 haplotype associates with reduced cortical surface area in humans in two independent populations. Proc Natl Acad Sci USA. 106:15483-15488.

Kremen WS, Franz CE, Lyons MJ. 2013. VETSA: the Vietnam Era Twin Study of Aging. Twin Res Hum Genet. 16:399-402.

Kremen WS, Prom-Wormley E, Panizzon MS, Eyler LT, Fischl B, Neale MC. et al. 2010. Genetic and environmental influences on the size of specific brain regions in midlife: The VETSA MRI study. Neuroimage, 49:1213-1223.

Kremen WS, Thompson-Brenner H, Leung YM, Grant MD, Franz CE, Eisen SA, et al. 2006. Genes, environment, and time: The vietnam era twin study of aging (VETSA). Twin Res Hum Genet. 9:1009-1022.

Lyons MJ, York TP, Franz CE, Grant MD, Eaves LJ, Jacobson KC. et al. 2009. Genes determine stability and the environment determines change in cognitive ability during 35 years of adulthood. Psychol Sci. 20:1146-1152. 
Marioni RE, Batty GD, Hayward C, Kerr SM, Campbell A, Hocking LJ. et al. 2014. Common genetic variants explain the majority of the correlation between height and intelligence: the generation Scotland study. Behav Genet. 44:91-96.

McGrevy DF, Knouse SB, Thompson RA. 1974. Relationships among an individual intelligence test and two air force screening and selection tests. ( No. Technical Report AFHRL-TR-74-25). San Antonio, TX: Personnel Research Division, Air Force Human Resources Laboratory, Brooks Air Force Base.

Neale, M. C., Boker, S. M., Xie, G., \& Maes, H. H. 2004. Mx: Statistical modeling. (6th ed.). Richmond, VA: Department of Psychiatry, Medical College of Virginia.

Neale MC, Cardon LR. 1992. Methodology for genetic studies of twins and families. Dordrecht, The Netherlands: Kluwer Academic Publishers.

O'Leary DD, Chou SJ, Sahara S. 2007. Area patterning of the mammalian cortex. Neuron. 56:252269.

Pakkenberg B, Gundersen HJ. 1997. Neocortical neuron number in humans: Effect of sex and age. The Journal of Comparative Neurology, 384:312-320.

Panizzon MS, Fennema-Notestine C, Eyler LT, Jernigan TL, Prom-Wormley E, Neale M, et al. 2009. Distinct genetic influences on cortical surface area and cortical thickness. Cereb Cortex, 19: 2728-2735. 
Panizzon MS, Vuoksimaa E, Spoon KM, Jacobson KC, Lyons MJ, Franz CE. et al. 2014. Genetic and Environmental Influences of General Cognitive Ability: Is g a valid latent construct? Intelligence. 43:65-76.

Rakic P. 2009. Evolution of the neocortex: A perspective from developmental biology. Nat Rev Neurosci. 10:724-735.

Jalil Razavi M, Zhang T, Liu T, Wang X. 2015. Cortical Folding Pattern and its Consistency Induced by Biological Growth. Sci Rep. 5:14477.

Ritchie SJ, Booth T, Valdés Hernández MD, Corley J, Maniega SM, Gow AJ, et al. 2015. Beyond a bigger brain: Multivariable structural brain imaging and intelligence. Intelligence. 51:47-56.

Schoenborn CA, Heyman KM. 2009. Health characteristics of adults aged 55 and over: United states 2004-2007. National Health Statistics Reports no. 16. MD: Hyatsville: National Center for Health Statistics. http://www.cdc.gov/nchs/data/nhsr/nhsr016.pdf

Schnack HG, van Haren NE, Brouwer RM, Evans A, Durston S, Boomsma DI. et al. 2015. Changes in thickness and surface area of the human cortex and their relationship with intelligence. Cereb Cortex. 25:1608-1617.

Shaw P, Greenstein D, Lerch J, Clasen L, Lenroot R, Gogtay N. et al. 2006. Intellectual ability and cortical development in children and adolescents. Nature. 440:676-679. 
Tallinen T, Chung JY, Biggins JS, Mahadevan L. 2014. Gyrification from constrained cortical expansion. Proc Natl Acad Sci USA. 111:12667-12672.

Van Essen DC, Dierker DL. 2007. Surface-based and probabilistic atlases of primate cerebral cortex. Neuron. 56:209-225.

Vuoksimaa E, Panizzon MS, Chen CH, Fiecas M, Eyler LT, Fennema-Notestine C. et al. 2015. The genetic association between neocortical volume and general cognitive ability is driven by global surface area rather than thickness. Cereb Cortex. 25:2127-2137.

Wierenga LM, Langen M, Oranje B, Durston S. 2014. Unique developmental trajectories of cortical thickness and surface area. Neuroimage. 87:120-126.

Winkler AM, Kochunov P, Blangero J, Almasy L, Zilles K, Fox PT. et al. 2010. Cortical thickness or grey matter volume? the importance of selecting the phenotype for imaging genetics studies. Neuroimage. 53:1135-1146.

Zacks JM. 2008. Neuroimaging studies of mental rotation: a meta-analysis and review. J Cogn Neurosci. 20:1-19.

Zilles K, Palomero-Gallagher N, Amunts K. 2013. Development of cortical folding during evolution and ontogeny. Trends Neurosci. 36(5):275-84. 
Table 1. Trivariate model fitting results for GCA, relative SA, and relative CT.

\begin{tabular}{|c|c|c|c|c|c|}
\hline Model & -211 & LRT & df & $\Delta \mathrm{df}$ & p-value \\
\hline 1. ACE-ACE-ACE Cholesky & 4074.984 & & 1524 & & \\
\hline 2. ACE-AE-AE & 4075.247 & 0.263 & 1529 & 5 & 0.998 \\
\hline 3. ACE-AE-AE, & 4087.564 & 12.317 & 1530 & 1 & 0.001 \\
\hline \multicolumn{6}{|l|}{ SA-GCA $r_{p}=$ CT-GCA $r_{p}$} \\
\hline 4. ACE-AE-AE, & 4082.551 & 7.304 & 1530 & 1 & 0.007 \\
\hline \multicolumn{6}{|l|}{ SA-GCA $r_{g}=$ CT-GCA $r_{g}$} \\
\hline 5. ACE-AE-AE, & 4077.435 & 2.188 & 1530 & 1 & 0.139 \\
\hline SA-GCA $r_{e}=$ CT-GCA $r_{e}$ & & & & & \\
\hline
\end{tabular}

Note. Model 1 is the comparison model for Model 2; Model 2 is the comparison model for Models

3-5. $\mathrm{A}=$ additive genetic variance $\mathrm{C}=$ common environmental variance; $\mathrm{E}=$ unique environmental variance; $r_{p}=$ phenotypic correlation; $r_{g}=$ genetic correlation; $r_{e}=$ environmental correlation; GCA = general cognitive ability; $\mathrm{SA}=$ cortical surface area; $\mathrm{CT}=$ cortical thickness; $-211=-2 \log$ likelihood; LRT = likelihood-ratio $\chi^{2}$-test; $\mathrm{df}=$ degrees of freedom; $\Delta \mathrm{df}=$ change in degrees of freedom. 
Table 2. Correlations between general cognitive ability (GCA) and the relative cortical surface area (SA) and thickness (CT) high-expanded - low expanded difference scores.

\section{GCA}

\section{$\underline{\text { Phenotypic correlations }}$}

Surface area

$.15(.05 ; .24)$

Thickness

$-.11(-.20 ;-.01)$

\section{Genetic correlations}

Surface area

Thickness

$\underline{\text { Unique environmental correlations }}$

Surface area

$.03(-.13 ; .19)$

Thickness

$-.15(-.31 ; .02)$

Note. Relative SA difference score $=($ SA of high-expanded ROI - SA of low-expanded ROI $) /$ total

SA. Relative CT difference score $=$ mean CT of high-expanded ROI - mean CT of low-expanded

ROI (see Fig. 1). Statistically significant ( $\mathrm{p}<.05)$ correlations are in bold $(95 \%$ confidence intervals in parentheses). Correlations are derived from the Model 2 in Table 1. 
Figure legends

Fig. 1. Desikan et al. (2006) regions-of-interest included in high-expanded and low-expanded cortices. High-expanded cortex (inside yellow boundaries) and the remaining low-expanded cortex correspond to high-low-expanded/lightly-heavily-myelinated cortex depicted in published studies (Hill et al., 2010; Glasser and Van Essen, 2011; Chaplin et al., 2013; Amlien et al., 2014; Fjell et al. 2014; Fjell et al. 2015) . High-expanded cortex includes (total of 15 Desikan ROIs): superior frontal gyri, middlefrontal gyri (rostral and caudal portions), inferior frontal gyri (pars opercularis, pars triangularis, pars orbitalis), frontal pole, middle temporal gyri, inferior temporal gyri, banks of the superior temporal sulcus, inferior parietal cortex, supramarginal gyri, anterior cingulate (rostral and caudal portions), posterior cingulate. Low-expanded cortex includes (total of 18 Desikan ROIs): orbital frontal cortex, medial orbital frontal cortex, precentral gyri, post central gyri, superior temporal gyri, transverse temporal, superior parietal cortex, lateral occipital cortex, paracentral lobule, precuneus, retrosplenial cortex, entorhinal gyri, parahippocampal gyri, temporal pole, lingual gyri, fusiform gyri, cuneus, pericalcarine cortex.

Fig. 2. Vertex-wise maps of cortical surface area-GCA correlations and cortical thickness-GCA correlations without accounting for global effects. SA = surface area; CT = cortical thickness. Shown from left to right in each row are left lateral, left medial, right lateral, and right medial views.

Fig. 3. Vertex-wise maps of relative regional cortical surface area-GCA correlations (relative to total surface area) and relative regional cortical thickness-GCA correlations (relative to mean thickness). SA = surface area; $\mathrm{CT}=$ cortical thickness. Shown from left to right in each row are left lateral, left medial, right lateral, and right medial views. 


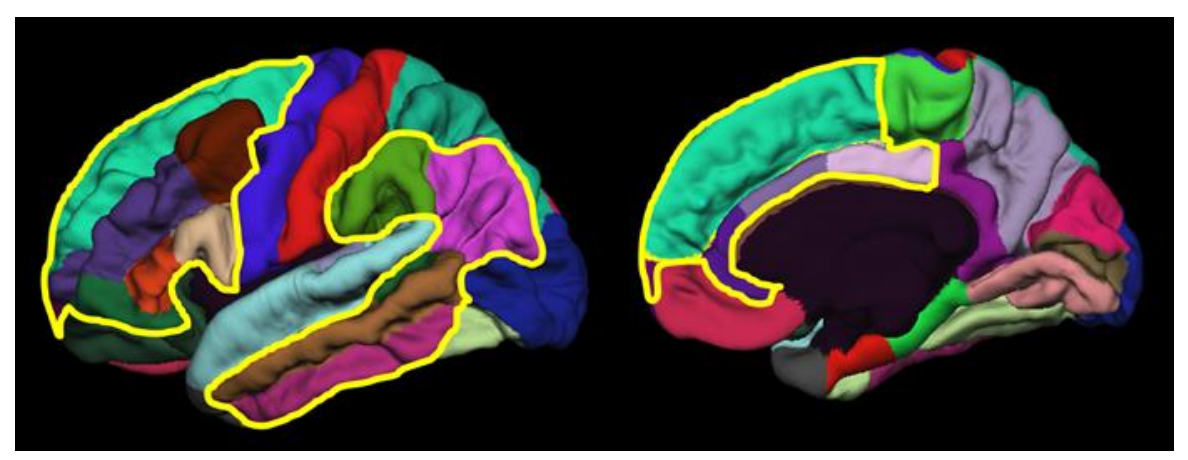




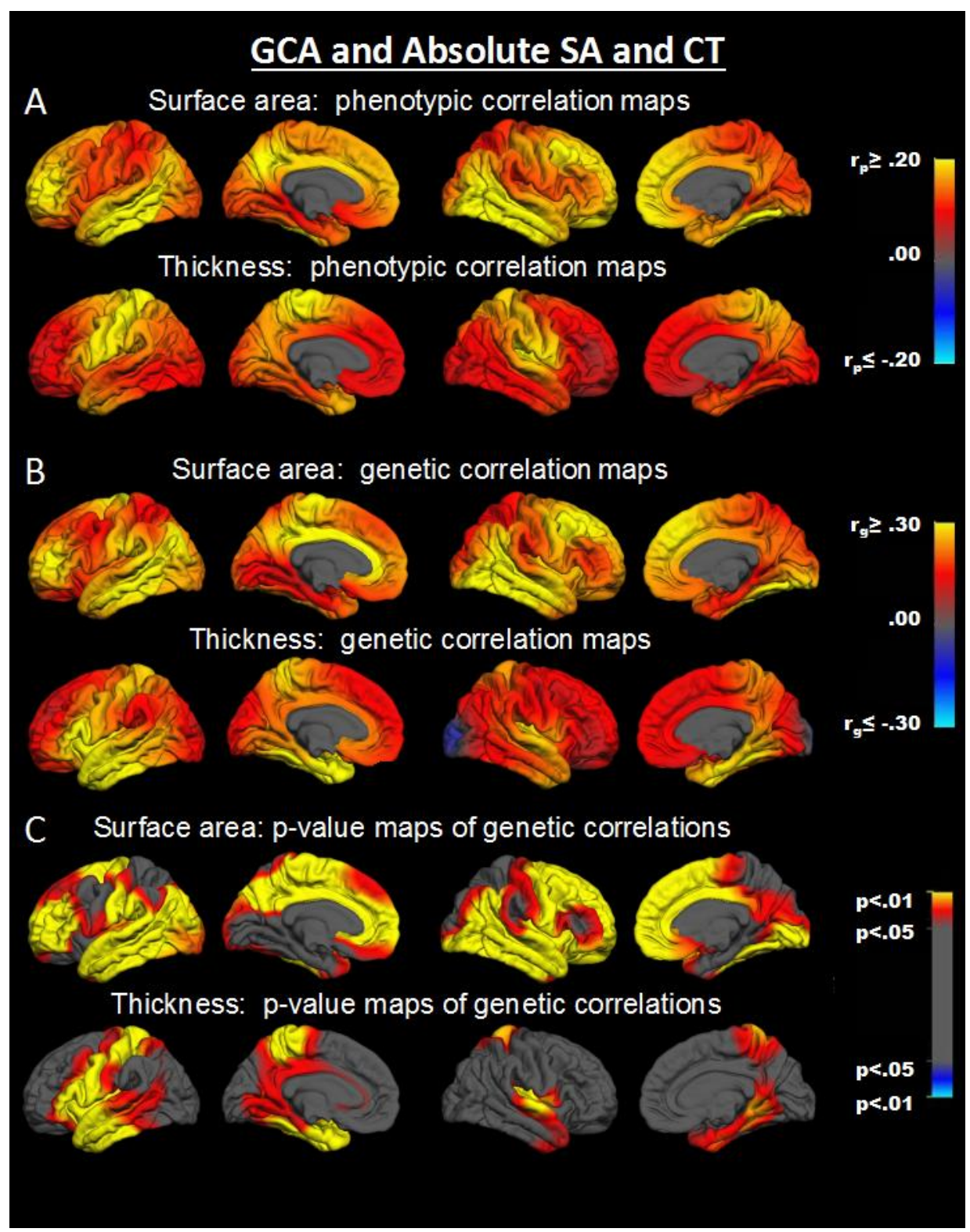




\section{GCA and Relative SA and CT}

\section{A Surface area: phenotypic correlation maps}
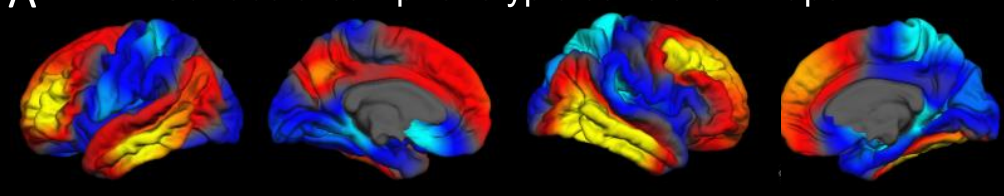

$r_{p} \geq .10$

Thickness: phenotypic correlation maps
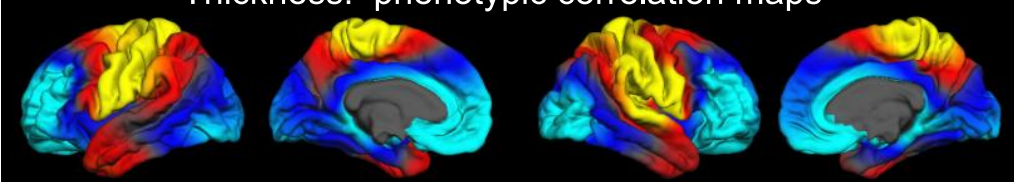

B Surface area: p-value maps of phenotypic correlations
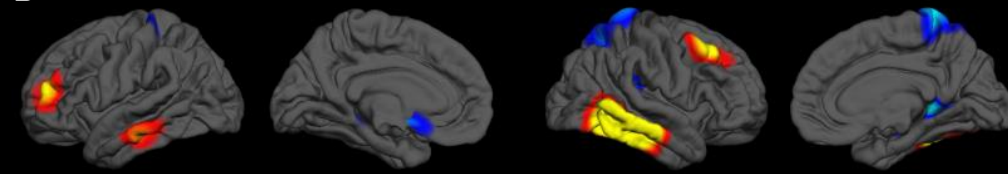

$p<.01$ p $<.05$

Thickness: p-value maps of phenotypic correlations
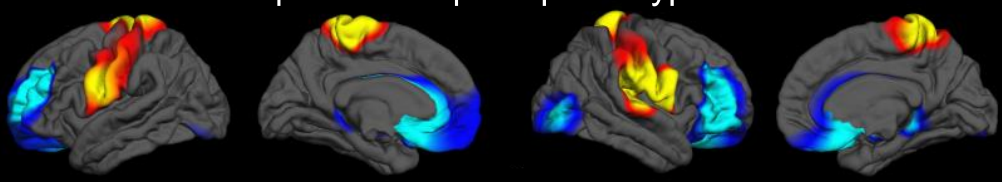

$p<.05$ $p<.01$

C

Surface area: genetic correlation maps
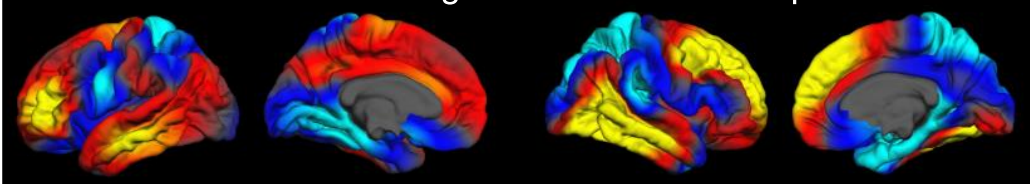

$r_{g} \geq .20$

.00

Thickness: genetic correlation maps
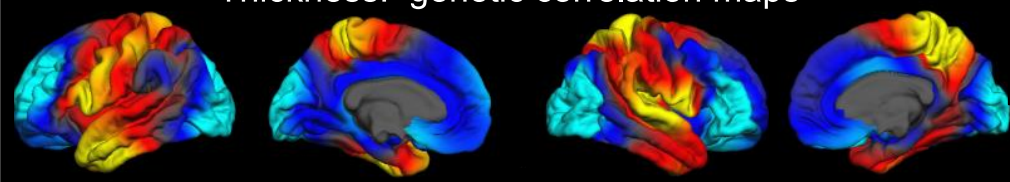

$r_{g} \leq-20$

D Surface area: $p$-value maps of genetic correlations
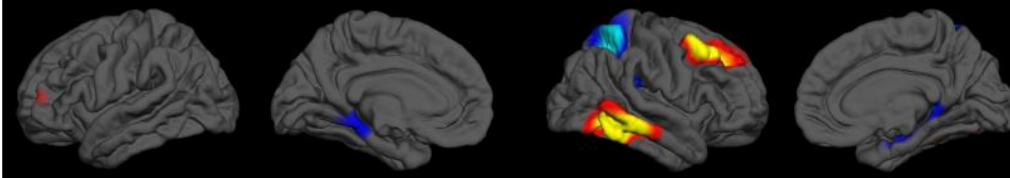

$p<.01$ $p<.05$

Thickness: p-value maps of genetic correlations
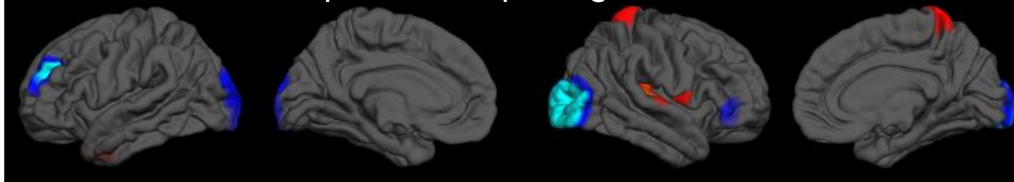